\title{
Supply the demand: Assessment of the feasibility of local non-urologists in relieving the burden of chronic indwelling catheters in a low-income country
}

Adam Bobrowski ${ }^{1}$; Madhur Nayan ${ }^{2}$; Olivier Heimrath ${ }^{2,3}$; Duncan Goche ${ }^{4}$; Enok Ludzu ${ }^{4}$; Rajiv K. Singal ${ }^{2,3}$

${ }^{1}$ Faculty of Medicine, University of Toronto, Toronto, ON, Canada; ${ }^{2}$ Division of Urology, Department of Surgery, University of Toronto, ON, Canada; ${ }^{3}$ Division of Urology, Michael Garron Hospital, Toronto, ON, Canada;

${ }^{4}$ Department of Surgery, Zomba Central Hospital, Zomba, Southeastern Region, Malawi

Acknowledgements: The authors thank Dignitas International and the University of Toronto's Urology Global Health Fund for their support, as well as the staff from MDH and ZCH for their assistance required for this initiative.

Cite as: Bobrowski A, Naya M, Heimrath O, et al. Supply the demand: Assessment of the feasibility of local non-urologists in relieving the burden of chronic indwelling catheters in a low-income country. Can Urol Assoc J 2020 October 27; Epub ahead of print. http://dx.doi.org/10.5489/cuaj.6567

Published online October 27, 2020

$* * *$

\section{Abstract}

Introduction: Despite the high prevalence rates of urinary retention in sub-Saharan Africa, regional deficiencies in urological care have culminated in inadequate medical management, and a backlog of urology cases. Our study examined the efficacy and safety of a surgical camp enlisting local non-urologists performing simple open prostatectomy on the rate of chronic catheter usage secondary to urinary retention.

Methods: We reported on a prospective case series of patients with chronic indwelling catheters who underwent open simple prostatectomy during a one-week urology camp in the Machinga District of Malawi. All operations were performed by a locally trained general surgeon and a clinical officer.

Results: Twenty-three (47.9\%) of 48 male patients with urinary retention assessed for eligibility for open simple prostatectomy were deemed eligible and underwent the procedure. Of the patients who underwent an open simple prostatectomy, histopathological findings demonstrated benign prostatic hyperplasia in 19 patients $(82.6 \%)$, while six patients $(26.1 \%)$ had coincidental malignancy. At postoperative followup, the entire cohort was catheter-free and reported regular sexual activity and the ability to return to work, while $87.0 \%$ noted improvements in social 
integration and 34.8\% cited higher self-esteem. Two patients required treatment for infection and one patient experienced fascial dehiscence. Two months following prostatectomy, all patients were catheter-free and able to void independently.

Conclusions: Local surgical practitioners without formal urology training can successfully perform open simple prostatectomy to relieve patients of chronic indwelling catheters and assist in addressing the disease burden in a low-resource setting.

\section{Introduction}

Urinary retention is a common urological problem varying in cause based on gender and age. In men, it can be secondary to benign prostatic hyperplasia (BPH) with management including pharmacologic and surgical interventions. In advanced BPH, surgery is the gold standard in patients with inadequate response to medical therapy or in cases of intractable urinary retention requiring catheterization. ${ }^{1}$

Technological advancements have transitioned surgical practice from open simple prostatectomy to minimally invasive techniques such as transurethral resection of the prostate (TURP) and laser enucleation or ablation as first-line interventions for BPH. ${ }^{1,2}$ However, a number of low- and middle-income countries (LMIC) do not have access to these surgical modalities, and generally lack the infrastructure to support and maintain the requisite instruments. Limited access to specialty surgical procedures due to a dearth of specialist training and inadequacies in the capacity to subsidize, sterilize and repair endourological equipment make the implementation of many standard urologic procedures an impossibility. ${ }^{3}$ Consequently, there is a discrepancy between the need for surgical care and its availability. In sub-Saharan Africa, an estimated 41 million additional procedures across all surgical subspecialties are required annually to satisfy current demand. ${ }^{4}$

The urology workforce in sub-Saharan Africa is exceedingly sparse, particularly in rural communities. ${ }^{5}$ Untreated urologic pathologies, namely BPH, urethral stricture, malignancy and urethral or ureteric trauma, constitute a large proportion of surgically-treatable disease burden. The prevalence of moderate-to-severe lower urinary tract symptoms (LUTS) is estimated at over 20.5 million people. ${ }^{5,6}$ Conditions that are easily amenable to surgical intervention remain insufficiently addressed.

In Malawi, there are currently no urologists to support a population exceeding 18.6 million people. ${ }^{7}$ Approximately $83.6 \%$ of the population inhabit rural communities, posing a particular challenge to healthcare accessibility. ${ }^{7}$ Urological procedures are primarily conducted in only four urban tertiary care centers in the country. ${ }^{8}$ Consequently, men presenting to rural clinics with urinary retention are often treated with indwelling urinary catheters that remain in place for years without a clear plan for definitive therapy. ${ }^{8}$ Chronic catheterization is associated 
with a myriad of complications, including infection, catheter blockage, leakage, pain, trauma, and an elevated risk of bladder stones, inflammation and malignancy. ${ }^{9}$

We sought to assess the efficacy and safety of a week-long surgical camp at the Machinga District Hospital (MDH) organized by local healthcare workers and enlisting local non-urologists performing simple open prostatectomy on the rate of chronic catheter usage secondary to urinary retention.

\section{Methods}

We reported on a prospective case series of patients in urinary retention managed with chronic indwelling catheters who underwent open simple prostatectomy during a week-long surgery camp in November 2018. This project was conducted as part of an expanding global surgery initiative within the Division of Urology at the University of Toronto. The Division of Urology outlined the framework and inclusion criteria for the surgical camp, and ethics approval was obtained from MDH.

Malawian healthcare is managed in a decentralized fashion. District healthcare logistics, financing, and provision is primarily directed by the District Health Management Teams (DHMT). The DHMTs are a conglomerate of healthcare providers, health policy and administrative workers. Clinical officers (CO) from the Machinga, Mangochi and Balaka DHMTs identified and recruited prospective patients for the camp. Men with chronic indwelling urinary catheters secondary to presumed BPH were deemed eligible for the study. All participants had suprapubic catheters. Participants were excluded if there was a clinical suspicion of prostate cancer, untreated urinary tract infection (UTI), or urinary retention secondary to a non-BPH etiology.

Informed consent was obtained from all participants prior to inclusion. Patient data was abstracted from clinical records. Patients were managed pre-operatively and post-operatively by clinicians and nurses from MDH. Vital signs, hemoglobin, blood group, urine cultures, duration of catheterization, and HIV status were recorded for all patients undergoing operation. The diagnosis of BPH was derived from digital rectal exam, transabdominal ultrasound, and past medical history, as assessed by camp-affiliated surgical staff. Prostate specific antigen (PSA) was not assessed as the MDH laboratory did not have the capacity to perform this assay.

Patient history was collected by the $\mathrm{CO}$ assisting with the operations and was utilized to assess for LUTS (frequency, urgency, nocturia, dysuria, weak stream, intermittency, straining and incomplete bladder emptying), and health-related quality of life (HRQOL). HRQOL parameters included: patient ability to ambulate and perform their vocation, urine leak, economic expenditures, and impact on self-esteem, socialization and sexual relationships. Post-operative follow-up was conducted at MDH at one week, two weeks, one month and two months. At follow-up, patient history would be re-assessed for LUTS and HRQOL. Catheter independence, ability to void, and urine stream were observed and recorded by the CO. Patient perspectives of 
stream strength were assessed on history. The unavailability of equipment to evaluate uroflowmetry prevented graded assessment of urine flow.

Prostatectomies were performed using a suprapubic technique under spinal anesthesia. All operations were performed by a single College of Surgeons of Eastern, Central and Southern Africa (COSECSA) certified staff general surgeon and a surgically trained CO from the Zomba Central Hospital $(\mathrm{ZCH})$. At the time of the camp's initiation, both practitioners had been in independent practice for three years. Through the COSECSA training program, the general surgeon received a 5-year surgical residency, augmented by instruction in the management of surgical emergencies pertaining to other subspecialties, including urology. ${ }^{10}$ The CO underwent a 2-year residency focused on general surgery. Neither the general surgeon nor CO received formal training in urology other than mentorship from visiting international urologists, although both became well acquainted with pelvic anatomy.

Dignitas International, a locally operational Toronto-based non-governmental organization, was responsible for logistical coordination of the surgical camp and the provision of materials required for the surgery. The University of Toronto's Urology Global Health Fund sponsored this initiative. Building capacity and developing sustainable surgical programs in LMICs through surgical training and mentorship has become a priority for the University of Toronto's Department of Surgery and Division of Urology. A staff urologist and urology resident affiliated with the University of Toronto provided mentorship on the performance of open prostatectomy at $\mathrm{ZCH}$ prior to the camp. No University of Toronto affiliated practitioners were directly involved with the operations or present at the camp. Specimens collected during the procedures were sent for histopathologic analysis at a private laboratory in Blantyre, Malawi. Pathology results were received approximately one week after delivery and communicated to patients.

\section{Results}

Forty-eight chronically catheterized patients recruited from three districts presented to the surgical camp at MDH. The majority of these patients were from Machinga District $(n=32)$, while the remainder originated from the Mangochi $(n=9)$ and Balaka $(n=7)$ Districts $(66.7 \%$, $18.8 \%$ and $14.6 \%$, respectively) (Figure 1). Pre-operative assessments demonstrated that 26 patients $(54.2 \%)$ were eligible for surgery. Of this cohort, nineteen $(86.4 \%)$ were deemed ineligible due to previously or newly diagnosed prostate cancer, two patients had positive urine cultures and symptomology consistent with a UTI $(9.09 \%)$, and one patient was not fit to undergo surgery due to a recent cerebrovascular infarction (4.55\%) (Table 1). Of the eligible candidates, 23 patients $(88.5 \%)$ underwent simple prostatectomy. Two patients were subsequently found to be ineligible due to previous orchiectomies for metastatic prostate cancer, and one patient was excluded as the procedure was aborted following the discovery of a large bladder mass intra-operatively. 
Patient demographic and clinical information is outlined in Table 1. The median age of patients undergoing simple prostatectomy was 70 years old. The majority of the patients originated from Machinga District (91.3\%). At presentation, 16 patients $(69.6 \%)$ were hypertensive, two patients $(8.70 \%)$ were anemic, and one patient $(4.35 \%)$ had HIV managed with antiretroviral therapy. Two patients reported frequent UTIs, while another patient was treated with pre-operative antibiotics due to painful micturition and purulent urethral discharge. One patient experienced abdominal pain and pruritus associated with his catheter.

Patient-reported HRQOL issues associated with chronic indwelling catheters were common. All participants cited catheter maintenance as a significant financial burden, primarily due to transportation costs to clinics. Additionally, an inability to perform field work and sexual dysfunction due to indwelling catheterization was reported by $95.7 \%$ and $87.0 \%$ of the cohort, respectively. Seven patients $(30.4 \%)$ described issues relating to catheter leakage and five patients described feelings of embarrassment and social isolation ( $21.7 \%$, respectively) relating to their chronic indwelling catheters (Table 2). All indwelling catheters were suprapubic. Median catheterization time was 24 months (IQR: 35.5) (Table 3).

Following suprapubic prostatectomy, patients were monitored at the MDH for a median of 7 days (IQR: 1). Clavien-Dindo grade II and IIIa complications were each experienced by one patient (4.35\%, respectively). One patient experienced fascial dehiscence at post-operative day 3 , which necessitated a return to the operating room for repair under regional anesthesia. While no blood transfusions were required intra-operatively, one patient was administered 500cc of packed red blood cells two weeks post-operatively due to a persistently low hemoglobin.

On histopathologic review, 19 patients $(82.6 \%)$ were found to have $\mathrm{BPH}$, while previously undiagnosed co-incidental prostate cancer was discovered in four patients (17.4\%). Two patients had Gleason 6 and Gleason 7 disease, respectively. Patients with co-incidental malignancy were treated according to local standards and underwent bilateral orchiectomies one month following prostatectomy. ${ }^{11}$ Of the remaining two patients, one patient had severe acute prostatitis and another patient had prostatic urethritis with ulceration. Interestingly, these patients were distinct from the two patients suffering from chronic UTIs pre-operatively.

At two-month post-operative follow-up, all patients were catheter independent. Nurses and the $\mathrm{CO}$ affiliated with the camp observed the ability of catheter-free patients to void independently. On history, twenty-three patients reported improvements to stream strength. Due to the inaccessibility of uroflowmetry equipment at the camp, graded urine flow rates were not established. The entire cohort reported regular participation in sexually activity in the postoperative period whereas $87.0 \%(n=20)$ of the cohort had complained of sexual dysfunction preoperatively. Two patients $(8.33 \%)$ specifically endorsed improved sexual performance. All patients noted the ability to return to work and perform occupational duties, 20 patients $(87.0 \%)$ reported improvements in social integration, and 8 patients $(34.8 \%)$ experienced improved selfesteem. One patient who suffered frequent UTIs pre-operatively reported a decrease in the 
occurrence of dysuria and infection. All patients reported a financial benefit from catheter removal as they no longer needed to pay out-of-pocket for catheter replacement or transportation to clinic. Since no validated tool was utilized to assess for LUTS and HRQOL in the pre- and post-operative period, the degree of improvements could not be quantified.

\section{Discussion}

Our report describes the first urologic surgery camp conducted in Malawi. This study sought to assess the feasibility of a surgical camp utilizing locally-trained practitioners with no formal training in urology performing simple open prostatectomy and its impact on the rate of chronic catheter usage. Despite a large burden of urologic pathology in this region, there are currently no urologists or established urology programs to serve Malawi's population. ${ }^{8}$ Consequently, open prostatectomy is performed by general surgeons and COs without extensive urologic training. ${ }^{12}$ Constraints to anesthetic services, inadequate surgical resources and expertise, and stagnant health systems infrastructure places a premium on preforming bellwether procedures rather than non-emergent operations. ${ }^{13}$

Medical management of BPH is practically unattainable in this region. Many essential medications are not readily available in public and private clinics and drug costs often exceed the statutory minimum daily wage, making pharmacologic therapeutic options unaffordable for many Malawian men. ${ }^{14}$

A previous study by our group described the diversity of urologic cases seen at $\mathrm{ZCH}$ in Malawi. ${ }^{8}$ Of particular interest, over a third of presentations were related to urinary retention, but less than $15 \%$ of these comprised surgical cases. ${ }^{8}$ A comparable prospective Ghanaian study found $\mathrm{BPH}$, urinary stricture and prostate cancer to be the most common etiologies of acute and chronic urinary retention. ${ }^{15}$ The sizable burden of urologic disease in Malawi compounded by the endemic inadequacy of trained specialists and infrastructure has resulted in men seeking care from rural clinics where chronic indwelling catheterization is the standard of care for urinary retention. ${ }^{8,16}$ Patients are catheterized without a final diagnosis or plan for long-term follow-up. Anecdotally, there are approximately 2000 men with chronic indwelling catheters in the Zomba District of Malawi alone. ${ }^{8,13}$ In our study, patients were catheterized for a median of 2 years and this duration of catheterization was similar to that reported by other studies from sub-Saharan Africa. ${ }^{16-21}$

All patients had suprapubic catheters, while patients in other referenced studies primarily utilized urethral catheters. ${ }^{17-22}$ Although the suprapubic route reduces the risk of urethral trauma and facilitates easier drainage and greater mobility, this approach negatively impacts self-esteem, body image and, to a lesser extent, sexual activity. ${ }^{23}$ In our study, $87.0 \%$ of patients reported impaired sexual functioning due to catheterization, emphasizing the well-established relationship between the LUTS of BPH and sexual dysfunction. ${ }^{24}$ Medical and surgical treatment of BPH addresses these concerns and enhances sexual wellbeing. 
The psychological impact of catheterization was profound in our population. Catheterrelated complaints included an inability to work (95.7\%), urine leak (30.4\%), social isolation (21.7\%), and embarrassment (21.7\%). Nearly half of a Nigerian cohort reported depressed mood as a consequence of indwelling catheterization. ${ }^{17}$

The financial burden of urinary catheters raised by our cohort has been noted in the literature. ${ }^{17-22}$ The estimated annual cost of indwelling catheter replacement in a Nigerian clinic was found to be over $\$ 50,000 .{ }^{19}$ In the short term, charitable platforms like surgery camps offer a viable means of containing costs and clearing the backlog of patients awaiting surgery. ${ }^{25,26}$ In the long term, Malawi will need to address shortages in the country's surgical and anesthetic workforce whilst optimizing infrastructure and preoperative triage. ${ }^{27}$ Nonetheless, patients may elect to maintain their catheters rather than pursue surgery due to extensive wait times, financial considerations, or culturally-related fears of operative intervention. ${ }^{4,18}$

Simple prostatectomy is an established treatment for BPH, but the utility of this procedure in the hands of local practitioners without formal urology training can be legitimately questioned. Our experience suggests this intervention has allowed study participants to remain catheter-free, with subjective improvement in multiple HRQOL parameters. Compared to other regional studies assessing simple prostatectomy to treat urinary retention, our study found lower mortality, complication and transfusion rates. ${ }^{17-22}$

While camps have been the most common method of foreign surgical assistance, there can be potential pitfalls to these well-meaning interventions. ${ }^{28,29}$ Primarily, these missions can promote dependence on ad hoc surgical programs rather than self-sufficiency. ${ }^{25}$ Secondly, the short-term nature of these projects can lead to precarious post-operative management. However, our project was disease-specific and urology focused, and the camp was run by local healthcare practitioners, with all operations performed by a Malawian general surgeon and CO. Nevertheless, the issue of long-term follow-up is currently being studied and the ultimate success of this camp model will determine whether the significant burden of urologic disease in Malawi can be safely remedied without resorting to investment in more specialized centers.

Currently, the bulk of TURPs in Malawi are performed at ZCH in conjunction with international surgeons. The training of domestic practitioners promotes sustained access to these curative interventions with the aim of fostering self-reliance. Task sharing, the practice of employing non-physicians to assume duties typically delegated to surgeons and anesthesiologists, has been common practice in sub-Saharan Africa. ${ }^{11,30,31}$ Surgically trained COs have proven to be cost-effective and comprise $92.7 \%$ of the surgical workforce in Malawian government hospitals. ${ }^{11,30-32}$ Outcomes of surgeries performed by COs, especially as applied to basic urologic procedures, have been shown to be similar to trained physicians. ${ }^{11,30,32}$

Dignitas International and the Global Surgery Program at the University of Toronto have taken steps to improve urological care in Malawi. These plans are focused on augmenting the technical and operational capacity of surgical departments in district hospitals and improving 
access to histopathologic services. To address the lack of urologic expertise in Malawi, the University of Toronto has funded the training of a Malawian surgical resident through the COSECSA program in Zambia with a view of an ultimate placement at $\mathrm{ZCH}$ as a urologist in 2020. There will be a concurrent investment in expanding anesthesia and endoscopic training at $\mathrm{ZCH}$ while additional efforts will focus on the engagement of general surgeons in urologic camps. An assessment of the long-term social, economic and medical impact of these policies will be necessary to validate this approach as a means of delivering urological care in resourcelimited settings.

This study presents several limitations. The urologic surgery camp at MDH has served to liberate men of their catheter dependency, but these results need to be objectively validated going forward. Post-operative follow-up demonstrated that open simple prostatectomy led to near uniform self-reported improvements in HRQOL measures and LUTS. Firstly, this information was collected primarily through patient history rather than evidence-based tools such as the International or Visual Prostate Symptom Score, which may have demonstrated a standardized response to treatment. Secondly, the lack of uroflowmetry equipment in this setting further complicates objective analysis. Nevertheless, this qualitative review has demonstrated improvements in HRQOL and urinary function by removing patient dependency on catheterization. Future studies of urine flow over time using available resources (for example, a graded cylinder and a stopwatch) are planned. Thirdly, detailed data of ineligible patients was not collected. The logistical challenges raised by the lack of permanent dedicated research staff, the relatively small numbers of patients spread over a sizeable geographical region, and a dearth of centralized laboratory and diagnostic facilities complicate follow-up. Patients who reported subjective improvements in their ability to urinate may be unmotivated to attend follow up appointments. Notwithstanding these shortcomings, it is important to note that one of the aims of this study was to allow local practitioners to manage all aspects of the camp in order to evaluate the sustainability of this program. Ultimately, the trade-off between robust reporting of data compatible with Western standards was balanced against therapeutic outcomes associated with a high level of functional improvement and patient satisfaction.

\section{Conclusions}

Our study has demonstrated that local general surgeons and COs without formal urological training can successfully perform open simple prostatectomy to relieve patients of long-term indwelling catheters secondary to chronic urinary retention. As such, non-urologists have the potential to be a vital component in addressing the high burden of urologic disease in a lowresource setting like Malawi. 


\section{References}

1. Nickel JC, Aaron L, Barkin J, et al. Canadian Urological Association guideline on male lower urinary tract symptoms/benign prostatic hyperplasia (MLUTS/BPH): 2018 update. Can Urol Assoc J 2018;12:303.

2. Hartung R. Transurethral prostatectomy (TURP): still the gold standard?. J Urol 1995;101:18.

3. Stothers L, Macnab A. Global implementation of advanced urological care: Policy implementation research. Can Urol Assoc J 2017;11:157.

4. Meara JG, Leather AJ, Hagander L, et al. Global Surgery 2030: Evidence and solutions for achieving health, welfare, and economic development. Surg 2015;158:3.

5. Campain NJ, MacDonagh RP, Mteta KA et al. Current challenges to urological training in sub-Saharan Africa. BJU Int 2015;116:316.

6. Bajunirwe F, Stothers L, Berkowitz J, et al. Prevalence estimates for lower urinary tract symptom severity among men in Uganda and sub-Saharan Africa based on regional prevalence data. Can Urol Assoc J 2018;12:E447.

7. OECD. The Future of Rural Youth in Developing Countries: Tapping the Potential of Local Value Chains, Development Centre Studies. OECD Publishing 2018.

8. Juvet T, Hayes JR, Ferrara S, et al. The burden of urological disease in Zomba, Malawi: A needs assessment in a sub-Saharan tertiary care center. Can Urol Assoc $J$ 2020;14:E6.

9. Murphy C, Cowan A, Moore K, et al. Managing long term indwelling urinary catheters. BMJ 2018;363:k3711.

10. Kakande I, Mkandawire N, Thompson MI. A review of surgical capacity and surgical education programmes in the COSECSA region. East Cent Afr J Surg 2011;16:6.

11. Kendig CE, Samuel JC, Tyson AF, et al. Cancer treatment in Malawi: a disease of palliation. World J Oncol 2013;3:142.

12. Gajewski J, Borgstein E, Bijlmakers L, et al. Evaluation of a surgical training programme for clinical officers in Malawi. Br J Surg 2019;106:e156.

13. Raveendran L, Bobrowski A, Singal RK. Men's health: a novel role for the urologist on the global health stage. BJU Int 2020;125:338.

14. Khuluza F, Haefele-Abah C. The availability, prices and affordability of essential medicines in Malawi: A cross-sectional study. PLoS One 2019;14:e0212125.

15. Yenli E, Aboah K, Sarpong K, et al. Acute and chronic urine retention among adults at the urology section of the Accident and Emergency Unit of Komfo Anokye Teaching Hospital, Kumasi, Ghana. Afr J Urol 2015;21:129.

16. Weiser TG, Regenbogen SE, Thompson KD, et al. An estimation of the global volume of surgery: a modelling strategy based on available data. Lancet 2008;372:139.

17. Abiola OO, Adeniyi SO, Williams-Abiola OT, et al. Quality of life and prevalence of depressive symptoms among patients on prolonged indwelling urinary catheters: A study from South west, Nigeria. Int J Med Med Sci 2016;8:96. 
18. Bello J, Ushie FA, Kuranga SA, et al. Prolonged use of indwelling urinary catheter following acute urinary retention in a tertiary care centre in sub-Saharan Africa: Causes, costs and concerns. Afr J Urol 2013;19:82.

19. Ikuerowo SO, Ogunade AA, Ogunlowo TO, et al. The burden of prolonged indwelling catheter after acute urinary retention in Ikeja - Lagos, Nigeria. BMC Urol 2007;7:16.

20. Kyei MY, Mensah JE, Morton B, et al. Surgical Management of Bph in Ghana: A Need to Improve Access to Transurethral Resection of the Prostate. East Afr Med $J$ 2012;89:241.

21. Ugare UG, Bassey IA, Udosen EJ, et al. Management of lower urinary retention in a limited resource setting. Ethiop J Health Sci 2014;24:329.

22. Ugwumba FO, Ozoemena OF, Okoh AD, et al. Transvesical prostatectomy in the management of benign prostatic hyperplasia in a developing country. Niger J Clin Pract 2014;17:797.

23. Chapple A, Prinjha S, Salisbury H. How users of indwelling urinary catheters talk about sex and sexuality: a qualitative study. Br J Gen Pract 2014;64:e364.

24. McVary K. Lower urinary tract symptoms and sexual dysfunction: epidemiology and pathophysiology. BJU Int 2006;97 Suppl 2:23.

25. Frankel JK, Murphy GP. International volunteerism and urethral stricture disease: a review. Transl Androl Urol 2018;7:659.

26. Shrime MG, Sleemi A, Ravilla TD. Charitable platforms in global surgery: a systematic review of their effectiveness, cost-effectiveness, sustainability, and role training. World J Surg 2015;39:10.

27. Gutnik LA, Dielman J, Dare AJ, et al. Funding flows to global surgery: an analysis of contributions from the USA. Lancet 2015;385Supp12:S51.

28. Prin M, Eaton J, Mtalimanja O, et al. High Elective Surgery Cancellation Rate in Malawi Primarily Due to Infrastructural Limitations. World J Surg 2018;42:1597.

29. Biyani CS, Campain N, Moore M, et al. Urolink supporting the development of urological services in Hawassa, Ethiopia. BJU Int 2019;123:917.

30. Ashengo T, Skeels A, Hurwitz EJH, et al. Bridging the human resource gap in surgical and anesthesia care in low-resource countries: a review of the task sharing literature. Hum Resour Health 2017;15:77.

31. Henry JA, Frenkel E, Borgstein E, et al. Surgical and anaesthetic capacity of hospitals in Malawi: key insights. Health Policy Plan 2015;30:985.

32. Beard JH, Oresanya LB, Akoko L, et al. Surgical task-shifting in a low-resource setting: outcomes after major surgery performed by nonphysician clinicians in Tanzania. World J Surg 2014;38:1398. 
Figures and Tables

Fig. 1. Patients included in this study originated from the Mangochi, Balaka, and Machinga Districts of Malawi. Zomba Central Hospital represents the tertiary care center for these regions.

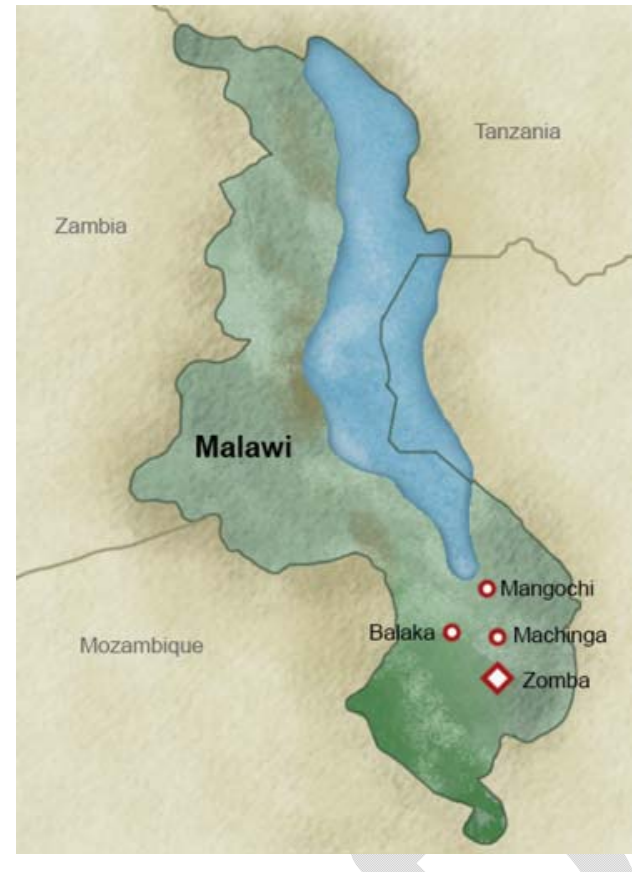

\begin{tabular}{|l|c|}
\hline Table 1. Baseline demographic and clinical information of simple prostatectomy patients \\
\hline Baseline demographic & n (\%) \\
\hline Number of eligible prostatectomy patients & $23(47.9 \%)$ \\
\hline Median age, years (IQR) & $70(14)$ \\
\hline District of origin & $21(91.3 \%)$ \\
\hline Machinga & $1(4.34 \%)$ \\
\hline Mangochi & $1(4.34 \%)$ \\
\hline Balaka & $22(45.8 \%)$ \\
\hline Ineligible patients & \\
\hline Reason for patient ineligibility & $19(86.4 \%)$ \\
\hline Prostate cancer & $2(9.09 \%)$ \\
\hline Urinary tract infection & $1(4.55 \%)$ \\
\hline Medically unfit for surgery & \\
\hline Baseline comorbidity & $16(69.6 \%)$ \\
\hline Hypertension & $2(8.70 \%)$ \\
\hline Anemia (defined as a hemoglobin $\leq 10 \mathrm{~g} / \mathrm{dl})$ & $1(4.35 \%)$ \\
\hline HIV & \\
\hline
\end{tabular}

IQR: interquartile range. 


\begin{tabular}{|l|c|}
\hline Table 2. Patient-reported health-related quality of life issues \\
\hline Patient-reported issues & $\mathbf{n}(\mathbf{\%})$ \\
\hline Financial challenges & $23(100.0 \%)$ \\
\hline Sexual dysfunction & $20(87.0 \%)$ \\
\hline Inability to perform field work & $22(95.7 \%)$ \\
\hline Catheter leakage & $7(30.4 \%)$ \\
\hline Social isolation & $5(21.7 \%)$ \\
\hline Embarrassment & $5(21.7 \%)$ \\
\hline Frequent urinary tract infections & $1(4.35 \%)$ \\
\hline
\end{tabular}

\begin{tabular}{|l|c|}
\hline Table 3. Duration of catheterization among patients receiving open simple prostatectomy \\
\hline Number of patients & Period of catheterization (months) \\
\hline 1 & 6 \\
\hline 1 & 7 \\
\hline 2 & 8 \\
\hline 1 & 12 \\
\hline 1 & 13 \\
\hline 1 & 15 \\
\hline 5 & 24 \\
\hline 1 & 25 \\
\hline 1 & 28 \\
\hline 2 & 36 \\
\hline 2 & 48 \\
\hline 1 & 51 \\
\hline 1 & 52 \\
\hline 1 & 60 \\
\hline 1 & 84 \\
\hline 1 & Not captured \\
\hline
\end{tabular}

